\title{
ANALYZING MIKROTIK BASED VRRP (VIRTUAL ROUTER REDUNDANCY PROTOCOL) IMPLEMENTATION ON HOMEGRID NETWORKS
}

\author{
Agustini Rodiah Machdi ${ }^{\left.a^{*}\right)}$ \\ a) Universitas Pakuan, Bogor, Indonesia \\ ${ }^{*}$ Corresponding Author : agustini.rodiah@unpak.ac.id
}

Article history: received 28 December 2018; revised 17 January 2019; accepted 25 January 2019

\begin{abstract}
Abstrak. Virtual Router Redundancy Protocol (VRRP) was developed to eliminate internal failures a single node gateway system, causing a network link to be disconnected. VRRP is capable provide redundant routing path link access. This time the research applied technology This VRRP is on a home network or building that utilizes the electricity installation network as LAN (Local Area Network) commonly called HomeGrid, and test it by doing QoS metric measurement. The QoS values obtained are then compared to the QoS standard on TIPHON as proof that network conditions are still in normal limits. The system is tested using 3 (three) schemes to get maximum results. Condition network using this VRRP system only requires very short downtime, when viewed from packet capture monitoring takes only 3 to 4 seconds in all schemes. Thus the technological capabilities of the VRRP system have been tested and are capable provide high availability links
\end{abstract}

Keywords: VRRP; Mikrotik; Router; JPerf; QoS; jitter

\section{INTRODUCTION}

Virtual Router Redundancy Protocol (VRRP) is the IETF standard provides a redundant router mechanism to increase network reliability there is. The VRRP process provides a failover dynamic responsible for continuing link to the Backup router (secondary) if the router Master (primary) has problems or power off [1]. VRRP provides benefits from the path defaults that have higher availability without requiring network devices to reconfigured if a failure occurs [2]. VRRP provides industry standard functions which is similar to Cisco's proprietary protocol Systems, Inc. named Hot Standby Router Protocol (HSRP), being a proprietary protocol from Digital Equipment Corporation, Inc. named IP Standby Protocol (IPSTB), and Protocol on Ownership of Foundry Networks named Fast Standby Router Protocol (FSRP) [3].

\section{RESEARCH METHODS}

QoS testing in this study using JPerf which is a tool application for measuring bandwidth and network link quality. Jperf can be Iperf graphic frontend written in Language Java programming. Network link that will measured is limited by two hosts run Jperf. The link quality is tested with the parameters as following [4]:

1. Latency/Delay (response time or RTT): can be measured by Ping command.

2. Jitter (variation of latency): can be measured with the Jperf UDP test.

3. Packet Loss or Datagram loss: can measured by the Jperf UDP test.

4. Throughput Bandwidth is measured through TCP test.
Difference between TCP (Transmission Control Protocol) and UDP (User Datagram Protocol) is that TCP uses process for checking that packages sent correctly to the recipient while with UDP packages sent without inspection but with the advantage of being faster than TCP.

Jperf uses TCP and UDP for produce measurement statistics different on a network link.

The following is the definition of the QoS parameter: Latency / Delay

According to Almes, G. Kalidindi, S. Zekauskas, M [5] Delay is delay time when the package is caused by the transmission process from another point that is the goal. Delay is obtained from time difference send between one TCP packet with other packages. The purpose of this measurement is to know the time needed package sent and arrived at the side receiver. This measurement too aims to find out the effects of every scenario that implemented. Jitter

According to Demichelis, C [6] Jitter is a variety of package arrivals, $\mathrm{p}$ this is caused by variations in queue length. Do measurement for find out the delay variations that occur and whether the burden is greater the traffic provided will also affects the quality of jitter.

Packet Loss

According to Almes, G. Kalidindi, S. Zekauskas, M [5] Packet loss is data that is lost at the time data packet delivery. The unit used is percent $(\%)$. The purpose of measuring Packet Loss is to find out how reliable technology used in maintaining a package to continue.

Throughput

According to Bradner, S [7] Throughput is the average speed data received by one node in the interval of observation certain. Throughput is actual bandwidth at that 
time we are connecting. The unit he has is the same as bandwidth is bps.

Measurement objectives for throughput this is to find out the reliability of the package arrived at the destination.

\section{System Implementation And Testing}

Implementation topology scheme on this study uses 3 schemes, namely [8]:

Scheme 1, testing and measurement QoS metric system VRRP on HomeGrid network with conditions home electricity without any load (no there is electrical equipment turned on), then do testing Redundancy of VRRP.

Scheme 2, testing and measurement QoS metric system VRRP on HomeGrid network by doing video streaming download activities, then do testing Redundancy of VRRP.

Scheme 3, testing and measurement QoS metric system VRRP on HomeGrid network for data transfer and streaming media (with conditions electrical equipment turned on), then redundancy testing VRRP.

\section{Measurement of all QoS on the network Homegrid without VRRP}

Measurement on this implementation, which is an initial condition, intended for test all QoS on the Homegrid network home electrical installation with no conditions electrical appliances are turned on and only use 1 unit of router mikrotik as gateway, router proxy is not configured as VRRP. Where is the QoS value (latency / delay, jitter, packet loss and throughput) later used as a comparison between conditions Homegrid network that uses 1 unit gateway router without VRRP with network Homegrid that uses 2 gateway routers which is configured as VRRP. Implementation diagram on Homegrid network this VRRP sign can be seen in Fig. 1 below.

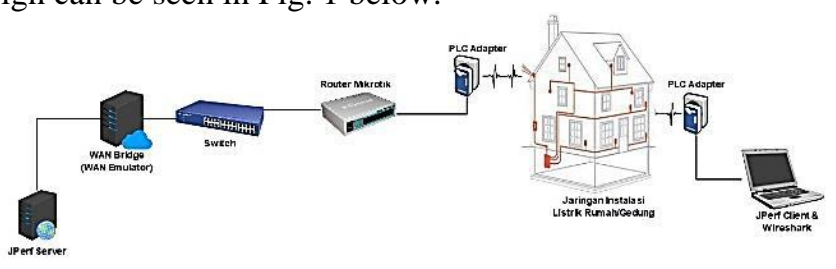

Figure 1 Diagram of homegrid network without VRRP

JPerf is designed to run as client / server application. To run the test, first have to prepare a JPerf server on the network, then run the client JPerf from another location on the network that will connected to the JPerf server.

\section{QoS Measurement on Networks VRRP Homegrid Scheme 1}

In the first scheme this corresponds to diagram in Figure 2 JPerf server is installed on the JPerf Server connected to the PC WAN Bridge, this WAN Bridge works for simulates a WAN connection for the router proxy gateway, then each router connected with a PLC / Homegrid device while the JPerf client is on duty collecting iPerf results data is a laptop connected with PLC / Homegrid through Cat 5e UTP cable. This first scheme was intended to test QoS on the Homegrid network electrical installation house with conditions without equipment electricity is turned on and uses 2 Mikrotik router unit as gateway, proxy the router is configured as VRRP.

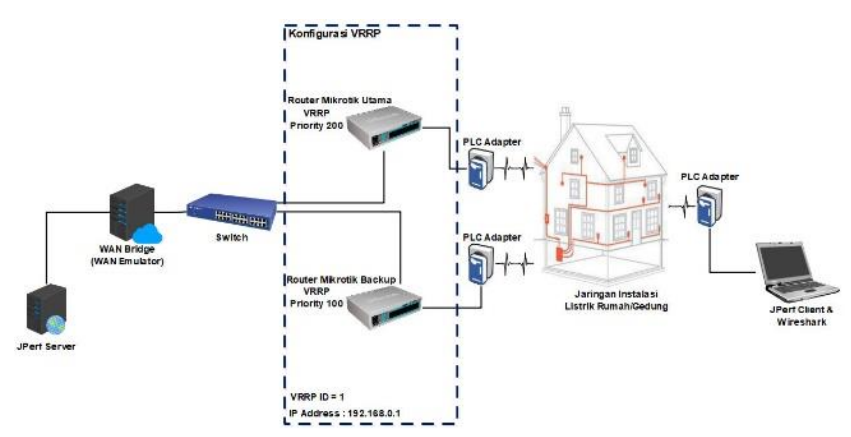

Figure 1. Network Diagram Scheme 1

Steps research for collect data on this first scheme as can be seen in Fig. 2 above as follows [9] :

1. Time used for this data collection is carried out for as long as 30 seconds

2. On the PC server is run JPerf Server

3. The client PC starts running JPerf Client

4. Then at the 10th second is done removal of Ethernet cable on the router the main proxy to run is the VRRP system already applied to run as as expected, namely VRRP failover the router backup mikrotik.

5. To see this done observation using the tool Wireshark application, following results from wireshark (Fig. 3) where it looks main router is offline and gateway switch to the backup router, visible in package number 13, this shows that the VRRP system managed to do a failover process when the main router is offline.

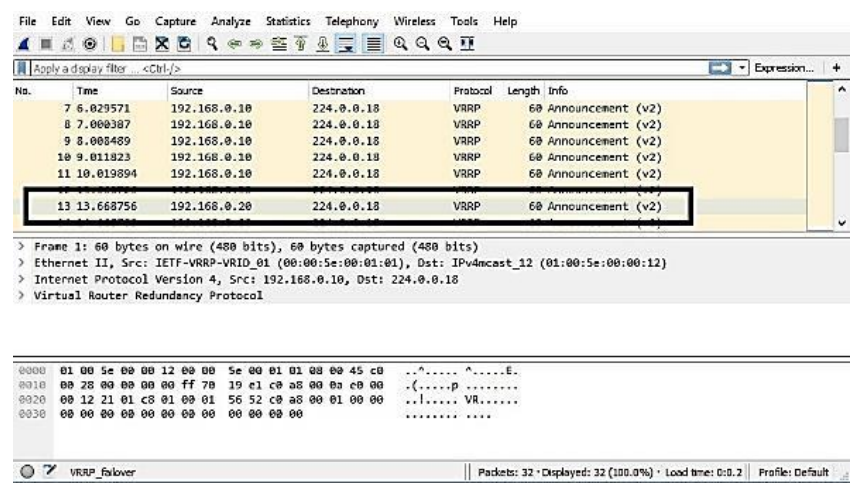

Figure 2. Display of observations on Wireshark Graphic Interface

6. Measuring using Jperf stopped after 30 seconds of time observations are reached.

7. QoS values obtained later recorded for later averaging its value. 


\section{QoS Measurement on Networks Homegrid VRRP Scheme 2}

For this second scheme, JPerf server installed on the connected JPerf Server PC on the WAN Bridge, use 2 units mikrotik router, then each router connected with a PLC / Homegrid device while the JPerf client is on duty collecting iPerf result data is a laptop connected with PLC / Homegrid through Cat 5e UTP cable according to the diagram on Fig. 3 This second scheme is intended to test QoS on the Homegrid network electrical installation house with conditions without equipment electricity is turned on, the network is loaded with streaming data and network traffic use 2 router mikrotik units as gateway, mikrotik router is configured as VRRP.

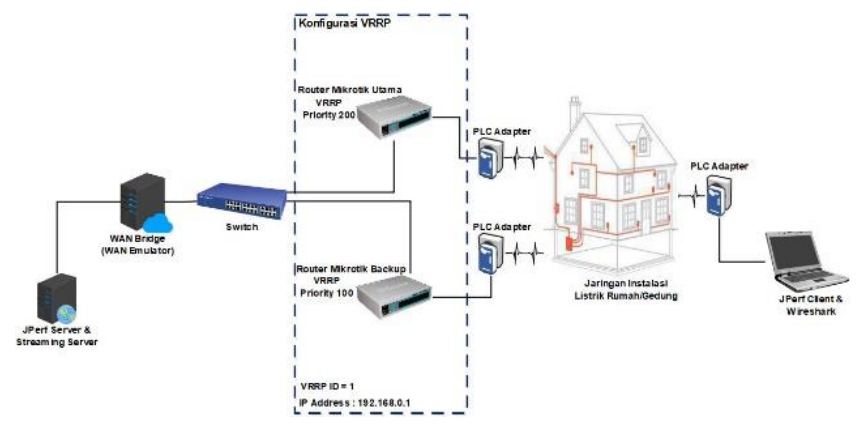

Figure 4. Network Diagram Scheme 2

Steps research for collect data on this second scheme as can be seen in Figure 4 above as follows :

1. Time used for this data collection is carried out for as long as 30 seconds

2. On the PC server is run JPerf Server and streaming server.

3. PC client does the download process video streaming from JPerf Server

4. Then PC client start run JPerf Client

5. Then at the 10th second is done removal of Ethernet cable on the router the main proxy to run is the VRRP system already applied to run as as expected, namely VRRP failover the router backup mikrotik.

6. Observation of VRRP failover process by using the application tool wireshark, the result of wireshark like seen in figure 3, the system VRRP successfully carried out the process failover on the main router offline.

7. Measuring using Jperf stopped after 30 seconds of time observations are reached. 8) QoS values obtained later recorded for later averaging its value.

\section{QoS Measurement on Networks VRRP Homegrid Scheme 3}

This third scheme is JPerf server installed on JPerf Server PC that is connected to the WAN Bridge, using 2 mikrotik router units, then each router is connected with PLC / Homegrid device while JPerf the client in charge of collecting results data iPerf is a laptop connected to it PLC / Homegrid through the appropriate UTP Cat 5e cable with the diagram in figure 5. This third scheme is intended to test QoS on the Homegrid network electrical installation home with all electrical equipment conditions turned on, the network is loaded with traffic streaming data and network using 2 Mikrotik router unit as gateway, proxy the router is configured as VRRP

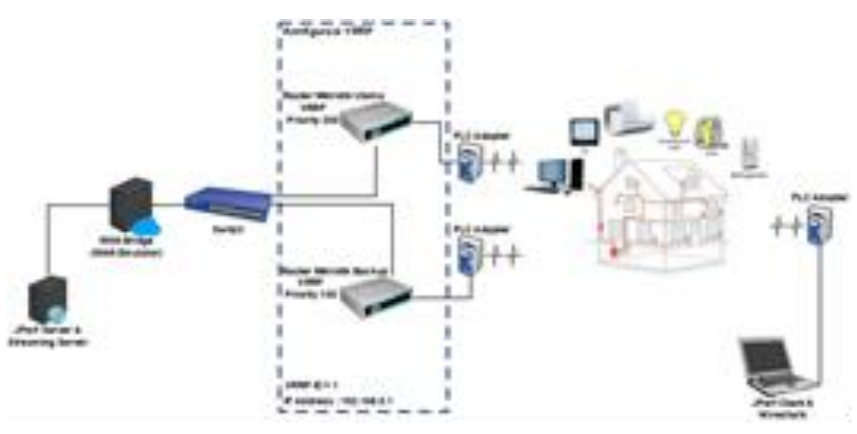

Figure 5. Network Diagram Scheme 3

Steps research for collect data on this second scheme as can be seen in Fig. 5 above as follows :

1. Time used for this data collection is carried out for as long as 30 seconds

2. On the PC server is run JPerf Server and streaming server.

3. All electrical equipment available turned on. 4) The PC client does the download process video streaming from JPerf Server

4. Then PC client start run JPerf Client

5. Then at the 10th second is done removal of Ethernet cable on the router the main proxy to run is the VRRP system already applied to run as expected, namely VRRP failover the router backup mikrotik.

6. Observation of VRRP failover process by using the application tool wireshark, the results of wireshark like as shown in Figure 3, the system VRRP successfully carried out the process failover on the main router offline.

7. Measuring using Jperf stopped after 30 seconds of time observations are reached.

QoS values obtained later recorded for later averaging its value.

\section{RESULTS AND DISCUSSION}

Data collection is done on home electricity networks with scenarios network implementation as discussed in the previous discussion. Electrical cable length connecting the two outlet points Homegrid is approximately 20 meters. Collection data is done by running JPerf application on both the server and client. From several tests that have been carried out then obtained the processed results and then the results are analyzed in this chapter.

\section{Measurement Results of Latency / Delay On All Schemes}

The JPerf application is run for 30 seconds and network interrupt unplug the cable from the main router / master is done in seconds 10 as a VRRP test then on each scheme is given a background load traffic is $0 \mathrm{Mbps}, 25$ Mbps, 50 Mbps, 75 Mbps. 
After data collection is complete and the results are averaged later obtained the latency / delay value for all schemes as shown on Table I as follows:

Table 1 Average value of latency / delay

\begin{tabular}{|c|c|c|c|c|c|}
\hline \multicolumn{7}{|c|}{ Delay (ms) } \\
\hline Skema & 0 Mbps & 25 Mbps & 50 Mbps & 75 Mbps & Keterangan \\
\hline TANPA VRRP & 4,10017 & 4,40038 & 4,48017 & 4,93219 & $\begin{array}{c}\text { Qualified berdasarkan } \\
\text { standar TIPHON }\end{array}$ \\
\hline Skema 1 & 4,68112 & 4,57991 & 4,78464 & 4,92271 & $\begin{array}{c}\text { Qualified berdasarkan } \\
\text { standar TIPHON }\end{array}$ \\
\hline Skema 2 & 9,36224 & 9,15982 & 9,56928 & 9,84542 & $\begin{array}{c}\text { Qualified berdasarkan } \\
\text { standar TIPHON }\end{array}$ \\
\hline Skema 3 & 11,36224 & 11,15982 & 12,56928 & 13,84542 & $\begin{array}{c}\text { Qualified berdasarkan } \\
\text { standar TIPHON }\end{array}$ \\
\hline
\end{tabular}

From table 1 data, average latency / delay then the results are formed into a graph like Fig. 6 below:

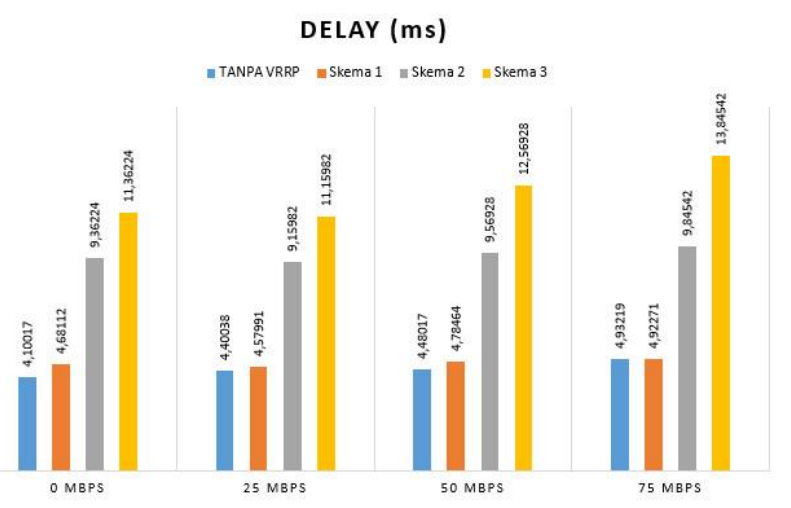

Figure 6. Graph of measurement of latency / delay in all schemes

\section{Analysis of Latency / Delay Measurement Results}

In the graph of the measurement results of Fig. 6 above, it looks for the best delay that is on the first scheme in each background load traffic. In this scenario the most visible delay small because there is no down time process inside it.

It's different from the delay status in the third scheme, in this scenario delay seen to increase more significantly due the process of streaming data traffic from the server to the laptop and vice versa with delay value at most $13,84542 \mathrm{~ms}$ still meets the standard of TIPHON with value very good under $150 \mathrm{~ms}$.

Delay in scheme two, not too significantly increased compared with the third scheme because of data traffic streaming. With thereby could concluded that the increase in traffic load then also increases the latency / delay value. On retrieve this latency / delay value, the value obtained is still in the range that can accepted from the standard set by TIPHON.

\section{Jitter Measurement in All Schemes}

The JPerf application is run for 30 seconds and network interrupt unplug the cable from the main router / master is done in seconds 10 as a VRRP test then on each scheme is given a background load traffic is $0 \mathrm{Mbps}, 25$ Mbps, 50 Mbps, 75 Mbps.

After data collection is complete and the results are averaged later obtained the results of the jitter value for the scheme one, scheme two and scheme three are displayed in Table II as follows:

Table 2. Average Value Of Jitter

\begin{tabular}{|c|c|c|c|c|c|}
\hline \multicolumn{7}{|c|}{ Jitter (ms) } \\
\hline Skema & 0 Mbps & 25 Mbps & 50 Mbps & 75 Mbps & Keterangan \\
\hline TANPA VRRP & 8,99865 & 9,18756 & 9,55873 & 9,77546 & $\begin{array}{c}\text { Qualified berdasarkan } \\
\text { standar TIPHON }\end{array}$ \\
\hline Skema 1 & 9,08112 & 9,57991 & 9,78464 & 9,92271 & $\begin{array}{c}\text { Qualified berdasarkan } \\
\text { standar TIPHON }\end{array}$ \\
\hline Skema 2 & 18,16224 & 19,15982 & 19,56928 & 19,84542 & $\begin{array}{c}\text { Qualified berdasarkan } \\
\text { standar TIPHON }\end{array}$ \\
\hline Skema 3 & 20,16224 & 21,15982 & 22,58158 & 23,87756 & $\begin{array}{c}\text { Qualified berdasarkan } \\
\text { standar TIPHON }\end{array}$ \\
\hline
\end{tabular}

From the data Table II, the average jitter is then converted into graphical form like that shown in the following Fig. 7:

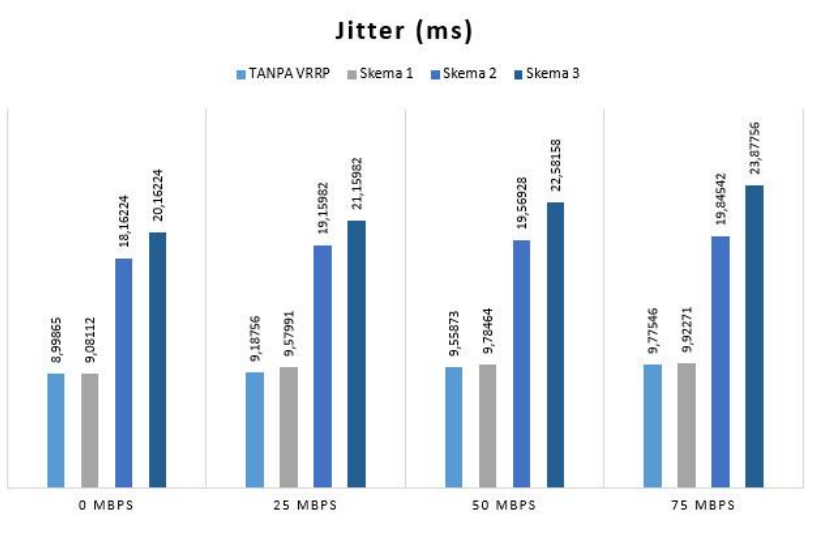

Figure 7. Jitter measurement graphic

\section{Analysis of Jitter Measurement Results}

The jitter parameter is used for measure network stability. From the results measurements taken on each The scheme shows that the delay increases accordingly with the burden of traffic provided. Getting more the amount of traffic load given jitter values are also getting bigger.

In the first scheme the jitter is getting more varies because traffic becomes denser by the advertisement package sent every 1 second on the same link.

Like the delay value, the most jitter value the height in Fig. 2 is obtained by the scheme three are affected by streaming data traffic with a value of $23.87756 \mathrm{~ms}$ in traffic variations $75 \mathrm{Mbps}$ but the value still meets the standard from TIPHON because the value obtained is still below 75 $\mathrm{ms}$. Whereas in the second scheme of values the greatest variation is also obtained traffic of $75 \mathrm{Mbps}$ with a value of $19.84542 \mathrm{~ms}$ and the smallest value by traffic variation 0 mbps with a value of $18.16224 \mathrm{~ms}$. 
This matter due to the value of the variation load Traffic is very influential on value jitter parameters are getting bigger the greater the amount of traffic provided also the jitter value issued.

Value obtained in this second scheme is still in good range with values that are still below standard value of 75 ms. In the first scheme the parameter values The resulting QoS jitter is not too large with the lowest value on traffic variations $0 \mathrm{mbps}$ is $9.08112 \mathrm{~ms}$ and the highest value obtained by 75 mbps traffic variations, namely $9,92771 \mathrm{~ms}$

This is because no interruptions occur in this system delay variations that occur from the laptop client towards the laptop server is not too large.

\section{Measurement of Thoughput in All Scheme}

The JPerf application runs for 30 seconds and network interrupt is revoked cable from the main router / master is done at 10 seconds as VRRP testing then in each scheme given background traffic load is $0 \mathrm{Mbps}, 25 \mathrm{Mbps}$, 50 Mbps, 75 Mbps.

After data collection is complete and the results averaged so that obtained throughput results for scheme one, scheme two and scheme three shown in Table III as follows:

Table 3. Average Throughput Value

\begin{tabular}{|c|c|c|c|c|}
\hline \multicolumn{5}{|c|}{ Throughput (Mbps) } \\
\hline Skema & 0 Mbps & 25 Mbps & 50 Mbps & 75 Mbps \\
\hline TANPA VRRP & 1,21422 & 1,19834 & 1,170083 & 1,00926 \\
\hline Skema 1 & 1,08112 & 1,07991 & 1,02464 & 1,00071 \\
\hline Skema 2 & 1,18923 & 1,18790 & 1,12710 & 1,10078 \\
\hline Skema 3 & 0,68923 & 0,68790 & 0,62710 & 0,60078 \\
\hline
\end{tabular}

From Table 3 data the average throughput then the results are graphed like shown in the following Fig. 8 :

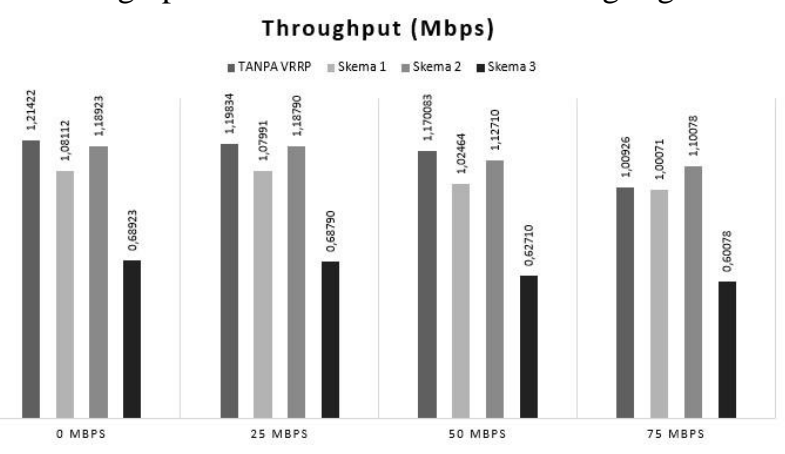

Figure. 8 Performance measurement throughput graphic

\section{Analysis of Throughput Measurement Results}

From the measurement results obtained that the greater the traffic load given, then the throughput value decreases accepted by the client side.
Throughput value best obtained by the first scheme with value of $1.08112 \mathrm{Mbps}$ for traffic variations of $0 \mathrm{Mbps}$ and the lowest value obtained with value of 1,00071 Mbps on 75 traffic variations Mbps. In the second scheme the value of throughput it is also best to get $0 \mathrm{Mbps}$ traffic variations with a value of $1.18923 \mathrm{Mbps}$ and the lowest obtained by $75 \mathrm{Mbps}$ traffic variations with a value of $1,10078 \mathrm{Mbps}$. While the best throughput value is in the third scheme is obtained in traffic variations $0 \mathrm{Mbps}$ with a value of $0.68923 \mathrm{Mbps}$ and value lowest in $75 \mathrm{Mbps}$ traffic variations with value of $0.60078 \mathrm{Mbps}$. The best value of the third the scheme is owned by the first scheme because it doesn't get interruptions on the network, in contrast to the third scheme where the throughput value tends to be more low due to the influence process electrical equipment and high data traffic. And it can be concluded that the more large variations of traffic provided then the smaller the throughput value will be obtained.

\section{Measurement of Packet Loss in All Scheme}

The JPerf application is run for 30 seconds and network interrupt unplug the cable from the main router / master is done in seconds 10 as a VRRP test then on each scheme is given a background load traffic is $0 \mathrm{Mbps}, 25$ Mbps, 50 Mbps, 75 Mbps. After data collection is complete and the results are averaged later obtained the value of packet loss for scenario 1 , scenario 2 and scenario 3 shown in Table IV as follows:

Table 4. Average Value Of Packet Loss

\begin{tabular}{|c|c|c|c|c|c|}
\hline \multicolumn{7}{|c|}{ Packet Loss (\%) } \\
\hline Skema & 0 Mbps & 25 Mbps & 50 Mbps & 75 Mbps & Keterangan \\
\hline TANPA VRRP & 0,00000 & 0,33146 & 0,41002 & 0,51218 & $\begin{array}{c}\text { Qualified berdasarkan } \\
\text { standar TIPHON }\end{array}$ \\
\hline Skema 1 & 0,00000 & 0,51214 & 0,52223 & 0,59101 & $\begin{array}{c}\text { Qualified berdasarkan } \\
\text { standar TIPHON }\end{array}$ \\
\hline Skema 2 & 4,44121 & 7,11162 & 7,99933 & 9,99991 & $\begin{array}{c}\text { Qualified berdasarkan } \\
\text { standar TIPHON }\end{array}$ \\
\hline Skema 3 & 9,88242 & 10,22324 & 11,99866 & 12,99982 & $\begin{array}{c}\text { Qualified berdasarkan } \\
\text { standar TIPHON }\end{array}$ \\
\hline
\end{tabular}

From the average packet loss (Table 4) then the results are converted into graphs as shown in Figure. 9 below:

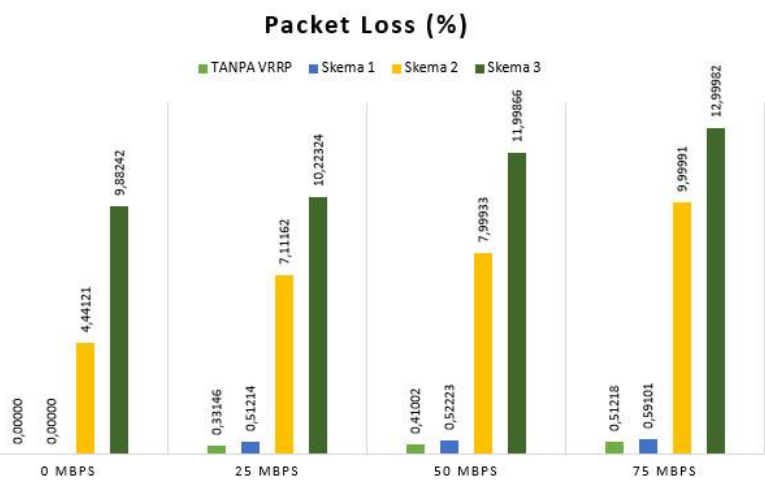

Figure 9. Measurement chart of Packet Loss 


\section{Analysis of Packet Loss Measurement Results}

From the measurement results it was found that the biggest packet loss occurs in the scheme third. This is due to the influence process electrical equipment and high data traffic with a value of $12.99982 \%$ on variations in traffic 75 Mbps which is in the standard TIPHON value it is still in the medium and acceptable.

While the value of packet loss the smallest in the third scheme is obtained by 0 Mbps traffic variation with a value of $9.88242 \%$. In the first scheme the value of packet loss the biggest is owned by $75 \mathrm{Mbps}$ traffic variations with a value of $0.59101 \%$ and packet loss value the lowest is the $0 \mathrm{Mbps}$ traffic variation with a value of $0 \%$.

Value the biggest packet loss in the scheme the second is owned by $75 \mathrm{Mbps}$ traffic variations with a value of $9.99991 \%$ and the lowest is owned by 0 Mbps traffic variation with value $4,44121 \%$. Can be concluded on value packet loss parameters are affected by the load traffic provided. Greater traffic what is given will be even greater packet loss parameter value.

\section{CONCLUSION}

The VRRP protocol is a solution a lot of fault tolerant networks used on LAN due convenience and have a fail-over time which is very short than the routing protocol other dynamic like RIP and also protocol This VRRP does not require modification network settings for hosts on LAN.

Measurement of QoS and packet values capture shows that the move from master router to the backup router at all does not affect the value of QoS, though when network traffic is high (when downloading video streaming is done), fail-over time which is still a second, or not perceived buffering process when download streaming video is done.

The results of delay measurement in the scheme without VRRP and the average VRRP scheme have difference of less than 0.5 seconds. As well from the measurement results of packet loss schemes without VRRP and schemes without VRRP the average has a difference of less than $1 \%$, so that all data transmission processes remain running as it should, and VRRP can be a solution of design telecommunication network in need network reliability from link breaks.

\section{REFERENSI}

[1] Towidjojo, Rendra. 2016. Mikrotik Kung Fu Kitab 3. Jakarta: Jasakom

[2] Wahana Komputer. 2014. Mudah Belajar Mikrotik Menggunakan Metode Virtualisasi. Yogyakarta: Andi

[3] Hardana., S.Kom. Irvanto,Ino. 2015. Belajar Mudah: Konfigurasi Routerboard Mikrotik RB-750. Yogyakarta: Andi

[4] Madcoms. 2017. Manajemen Sistem Jaringan Komputer Dengan Mikrotik RouterOs. Yogyakarta: Andi
[5] Almes, G., Kalidindi, S. and M. Zekauskas. 1999. A One-Way Packet Loss Metric for IPPM RFC 2680

[6] Demichelis, C. and P. Chimento. 2002. IP Packet Delay Variation Metric for IP Performance Metrics (IPPM). RFC 3393

[7] Bradner, S. 1997. Key words for use in RFCs to Indicate Requirement Levels. RFC 2119.

[8] Cohn,Ronald. Russell, Jesse. 2012. Iperf Paperback. New York: VSD Publisher

[9] Sukmaaji, Anjik S.Kom, Rianto S.kom. 2008. Jaringan Komputer Konsep Dasar Pengembangan Jaringan dan Keamanan Jarignan. Yogyakarta: Andi 